\title{
Differential Localization of Src Homology 2 Domain-Containing Protein Tyrosine Phosphatase Substrate- 1 and CD47 and Its Molecular Mechanisms in Cultured Hippocampal Neurons
}

\author{
Hiroshi Ohnishi, ${ }^{1 \star}$ Yuka Kaneko, ${ }^{1 \star}$ Hideki Okazawa, ${ }^{1}$ Motoaki Miyashita, ${ }^{1}$ Ryuji Sato, ${ }^{1}$ Akiko Hayashi, ${ }^{1}$ \\ Kazutoshi Tada, ${ }^{2}$ Shigekazu Nagata, ${ }^{2}$ Masami Takahashi, ${ }^{3}$ and Takashi Matozaki ${ }^{1}$ \\ ${ }^{1}$ Laboratory of Biosignal Sciences, Institute for Molecular and Cellular Regulation, Gunma University, Maebashi, Gunma 371-8512, Japan, ${ }^{2}$ Department of \\ Genetics, Osaka University Medical School, Suita, Osaka 565-0871, Japan, and ${ }^{3}$ Department of Biochemistry, Kitasato University School of Medicine, \\ Sagamihara, Kanagawa 228-8555, Japan
}

\begin{abstract}
Polarized localization of membrane proteins to axons or dendrites is important for a variety of neuronal functions, including neurite outgrowth and synaptogenesis during neural development. Src homology 2 domain-containing protein tyrosine phosphatase (SHP) substrate-1 (SHPS-1) and its ligand cluster of differentiation 47 (CD47), both of which are members of the Ig superfamily of proteins, are thought to constitute an intercellular communication system in the CNS, although the physiological functions of this CD47-SHPS-1 system remain unknown. To provide insight into these functions, we have now examined the localization of SHPS-1 and CD47 in cultured hippocampal neurons. Endogenous SHPS-1 was detected at the surface of both axons and dendrites, whereas endogenous CD47 was localized predominantly to the surface of dendrites. Forced expression of these two proteins confirmed their distinct localizations. The extracellular regions of SHPS-1 and CD47 were responsible, at least in part, for their axonal and dendritic localizations, respectively; however, the axonal localization of SHPS-1 was not mediated by any one of the three Ig domains in its extracellular region. Overexpression of SHPS-1 and CD47 in distinct neurons resulted in marked accumulation of these proteins at sites of contact between SHPS-1-expressing axons and CD47-expressing dendrites. Such contact sites exhibited an enlarged structure but did not contain the synaptic marker protein vesicle-associated membrane protein-2. These results suggest that differential localization of SHPS-1 and CD47 at axons and dendrites generates a directional intercellular communication system that potentially contributes to regulation of synaptogenesis and the formation of neural networks.
\end{abstract}

Key words: SHPS-1; CD47; Ig superfamily; axon; dendrite; hippocampus

\section{Introduction}

Regulation of neuron-neuron or neuron-glial cell interactions in the CNS is required for various neuronal functions such as cell migration, neurite projection, and synaptogenesis, all of which contribute to the formation of neural networks (Murase and Schuman, 1999; Fields and Stevens-Graham, 2002). Various recognition molecules on the surface of neurons and surrounding

\footnotetext{
Received Sept. 8, 2004; accepted Jan. 20, 2005.

This work was supported by a grant-in-aid for Scientific Research on Priority Areas Cancer, a grant-in-aid for Scientific Research (B), and a grant-in-aid for Young Scientists (B) from, as well as the 21st Century Center of Excellence Program of, the Ministry of Education, Culture, Sports, Science, and Technology of Japan and by grants from the Novartis Foundation (Japan) for the Promotion of Science, Uehara Memorial Foundation, Brain Science Foundation, Nakajima Foundation, and Japan Research Foundation for Clinical Pharmacology. We thank C. F. Lagenaur, J. Miyazaki, M. Kataoka, and S. C. Fujita for reagents, as well as K. Tomizawa and H. Kobayashi for technical assistance.

*H.O. and Y.K. contributed equally to this work.

Correspondence should be addressed to Dr. Takashi Matozaki, Laboratory of Biosignal Sciences, Institute for Molecular and Cellular Regulation, Gunma University, 3-39-15 Showa-Machi, Maebashi, Gunma 371-8512, Japan. E-mail:matozaki@showa.gunma-u.ac.jp.

DOI:10.1523/JNEUROSCI.5173-04.2005

Copyright $\odot 2005$ Society for Neuroscience $\quad$ 0270-6474/05/252702-10\$15.00/0
}

glia participate in such regulation during brain development. These recognition molecules include proteins that contain Ig-like domains, fibronectin type III-like domains, or epidermal growth factor domains in their extracellular regions (Kamiguchi and Lemmon, 2000), and some of them are localized differentially in axons or dendrites. Such polarized localization of recognition molecules is important for directional signaling between these neurites (Jareb and Banker, 1998; Winckler and Mellman, 1999).

Src homology 2 domain-containing protein tyrosine phosphatase (SHP) substrate-1 (SHPS-1), also known as SIRP $\alpha$ (Kharitonenkov et al., 1997), is a receptor-like transmembrane protein that we originally identified as a substrate for the protein tyrosine phosphatases SHP-1 and SHP-2 (Fujioka et al., 1996). The extracellular region of SHPS-1 comprises three Ig-like domains, and its cytoplasmic region contains four putative tyrosine phosphorylation sites that serve as binding sites for the Src homology 2 domains of SHP-1 and SHP-2 (Fujioka et al., 1996; Kharitonenkov et al., 1997). SHPS-1 was also previously identified as a brain membrane protein designated p84 (Chuang and 
Lagenaur, 1990) or BIT (Ohnishi et al., 1996). Indeed, SHPS-1 is particularly abundant in the CNS, and its expression is markedly associated with synapse-rich areas such as the molecular layer and synaptic glomeruli of the cerebellum, the plexiform layers of the retina, and the hippocampus (Chuang and Lagenaur, 1990; Comu et al., 1997). SHPS-1 undergoes tyrosine phosphorylation in cultured neurons in response to brain-derived neurotrophic factor or nerve growth factor (Ohnishi et al., 1999), and it is implicated in the regulation of the promotion of neuronal survival by brain-derived neurotrophic factor (Araki et al., 2000).

Cluster of differentiation 47 (CD47), also named integrinassociated protein, is a ligand for SHPS-1 (Jiang et al., 1999; Seiffert et al., 1999). This protein, which was originally identified in association with the integrin $\alpha \mathrm{v} \beta 3$, is also a member of the Ig superfamily, possessing a V-type Ig-like extracellular domain, five putative membrane-spanning segments, and a short cytoplasmic tail (Brown and Frazier, 2001). One of four alternatively spliced isoforms of CD47 (form 4) is predominantly expressed in the brain (Reinhold et al., 1995). CD47 and SHPS-1 appear to constitute a cell-cell communication system, and this CD47SHPS-1 system has been shown to contribute to the negative regulation of phagocytosis in macrophages (Oldenborg et al., 2000 ), to tethering of apoptotic cells to phagocytes (Tada et al., 2003), and to inhibition of cell migration by cell-cell contact (Motegi et al., 2003).

Through its interaction with SHPS-1, CD47 also promotes neurite or filopodium formation in N1E-115 neuroblastoma cells or cultured neurons (Miyashita et al., 2004). Although its physiological functions in the CNS remain unknown, these observations implicate the CD47-SHPS-1 system in the regulation of neurite extension and synaptogenesis. To gain insight into the role of the CD47-SHPS-1 system in neurons, we have now investigated the subcellular localization of endogenous and ectopic SHPS-1 and CD47 in cultured hippocampal neurons as well as the molecular mechanisms responsible for such localization.

\section{Materials and Methods}

Antibodies. Hybridoma cells that produce a rat monoclonal antibody (mAb) to mouse SHPS-1 (P84) were kindly provided by C. F. Lagenaur (University of Pittsburgh School of Medicine, Pittsburgh, PA); the mAb was purified from serum-free culture medium of the cells by affinity chromatography with protein G-Sepharose 4 Fast Flow (Amersham Biosciences, Piscataway, NJ). A rat mAb to mouse CD47 (MIAP301) was obtained from PharMingen (San Diego, CA). A hamster mAb to mouse SHPS-1 (FG2) was generated and prepared as described previously (Tada et al., 2003). A mouse mAb to phosphorylated neurofilaments (SMI31) was obtained from Sternberger Monoclonal (Lutherville, MD), a mouse $\mathrm{mAb}$ to microtubule-associated protein 2 (MAP2) was obtained from NeoMarkers (Fremont, $\mathrm{CA}$ ), and a mouse $\mathrm{mAb}$ to postsynaptic density protein-95 (PSD-95) was obtained from Upstate Biotechnology (Lake Placid, NY). Rabbit polyclonal antibodies to vesicle-associated membrane protein-2 (VAMP-2) were generated as described previously (El Far et al., 1995). A mouse mAb to synaptophysin (171B5) was kindly provided by S. C. Fujita (Mitsubishi Kagaku Institute of Life Sciences, Tokyo, Japan). A mouse mAb to human CD8 was obtained from eBioscience (San Diego, CA). A mouse mAb to the Myc epitope tag (9E10) was purified from the culture supernatant of hybridoma cells. Cyanine 3 (Cy3)-conjugated goat antibodies to mouse, rat, or hamster IgG and aminomethylcoumarin-conjugated goat antibodies to rabbit IgG were obtained from Jackson ImmunoResearch (West Grove, PA). Alexa 488conjugated goat antibodies to mouse, rat, or rabbit IgG were purchased from Molecular Probes (Eugene, OR).

Expression vectors for neurons. Expression vectors for mouse CD47, CD47 $\Delta$ Cterm, and CD8-CD47MMS were constructed as described previously (Miyashita et al., 2004). A cDNA fragment encoding full-length mouse CD47 tagged with the Myc epitope at its $\mathrm{COOH}$ terminus (mCD47myc) was generated by PCR with mouse CD47 (form 4) cDNA as the template and an antisense primer containing the DNA sequence for the Myc epitope at its 5' end. The mCD47myc cDNA was subcloned into the eukaryotic expression vector pCAGGS (Niwa et al., 1991), kindly provided by J. Miyazaki (Osaka University, Osaka, Japan), for expression of the encoded protein in cultured hippocampal neurons.

A plasmid encoding a green fluorescent protein (GFP)-VAMP-2 fusion construct was kindly provided by M. Kataoka (Shinsyu University, Nagano, Japan). Rat $\beta$-actin cDNA was also subcloned into the pEGFP-C2 vector (Clontech, Palo Alto, CA) downstream of the DNA sequence for GFP. The DNA fragments encoding the GFP-VAMP-2 and GFP-actin fusion proteins were then excised and subcloned into pCAGGS.

A cDNA fragment encoding full-length mouse SHPS-1 tagged with the Myc epitope at its $\mathrm{COOH}$ terminus (mSHPS-1myc) was generated by $\mathrm{PCR}$ with an antisense primer containing the DNA sequence for the Myc epitope at its 5' end. The mSHPS-1myc cDNA, a full-length cDNA for mouse SHPS-1 (Yamao et al., 1997), and a cDNA encoding the mutant SHPS-1 $\Delta$ Cyto, which lacks almost the entire cytoplasmic region of SHPS-1 (amino acids 405-509) (Sato et al., 2003), were then each subcloned into pCAGGS. To generate an Ig-domain deletion mutant of SHPS- $1, \Delta V$, that lacks amino acids 29-147, we performed PCR with the expression vector for mSHPS-1myc as the template, the sense primer $5^{\prime}$-ACCAGCGCTTTTCTCAGGCTGAGTCACCTT-3', and the antisense primer 5'-ACCAGCGCTAAACCTTCTCCACCGGACCT-3'. The PCR product was then digested with Eco47III and self-ligated. We also constructed other Ig-domain deletion mutants, $\Delta \mathrm{C} 1 \# 1$ (lacking amino acids 148-253) and $\Delta \mathrm{C} 1 \# 2$ (lacking amino acids 254-365), essentially as described for $\Delta \mathrm{V}$ but with the sense primer $5^{\prime}$-ACCAGCGCTGGCGAGTACATAGACCTCG-3' and the antisense primer $5^{\prime}$-ACCAGCGCTTCACCCACCGTGAAGGTCAC- $3^{\prime}$ for $\triangle \mathrm{C} 1 \# 1$ and the sense primer $5^{\prime}$-ACCAGCGCTAACTCGGATGAAGTTAGACAG-3' and the antisense primer $5^{\prime}$-ACCAGCGCTAATAATGCTACCCACAACTGG-3' for $\Delta \mathrm{C} 1 \# 2$.

To generate a cDNA fragment encoding the chimeric protein CD8SHPS-1cp, we amplified cDNA sequences encoding the extracellular and transmembrane domains of human CD8 (amino acids 1-208) and the cytoplasmic domain of mouse SHPS-1 (amino acids 399-509) by PCR from the corresponding full-length cDNAs. We also generated a cDNA encoding CD8- $\Delta$ Cyto, which lacks almost the entire cytoplasmic domain of CD8-SHPS-1cp (corresponding to amino acids 405-509 of mouse SHPS-1), by PCR with the cDNA for CD8-SHPS-1cp as the template. The DNA fragments for these CD8-SHPS- 1 chimeric proteins were also subcloned into pCAGGS. The nucleotide sequences of all amplified cDNAs were verified by sequencing with an ABI PRISM310 Genetic Analyzer (Applied Biosystems, Foster City, CA).

Primary culture of hippocampal neurons and transfection. Hippocampal neurons were isolated from mouse or rat embryos at gestational days 17-18 as described previously (Hayashi et al., 2002; Miyashita et al., 2004). The cells were plated on poly-D-lysine-coated cover glasses or plastic culture dishes at a density of $1 \times 10^{5}$ to $2 \times 10^{5} \mathrm{cells} / \mathrm{cm}^{2}$ for high-density culture or $5 \times 10^{3}$ cells $/ \mathrm{cm}^{2}$ for low-density culture. They were maintained in serum-free medium [Neurobasal medium (Invitrogen, Rockville, MD) supplemented with $0.5 \mathrm{~mm}$ glutamine and $\mathrm{B} 27$ supplement (Invitrogen)] at $37^{\circ} \mathrm{C}$ under a humidified atmosphere of $10 \% \mathrm{CO}_{2}$.

Neurons were transfected with expression vectors with the use of Lipofectamine 2000 (Invitrogen). In standard analyses, neurons were transfected after 10-12 d in vitro (DIV) and maintained for 2-4 d after transfection. In cotransfection experiments, vectors were mixed in advance of liposomal complex formation.

Immunofluorescence analysis. In standard immunofluorescence experiments, surface expression of SHPS-1 or CD47 was visualized in nonpermeabilized cells (without detergent). Neurons were fixed for 20-30 min at room temperature in PBS containing 4\% paraformaldehyde and then incubated for $60 \mathrm{~min}$ at room temperature in blocking solution (PBS containing 5\% goat serum). They were then incubated for $1 \mathrm{~h}$ at room temperature or overnight at $4^{\circ} \mathrm{C}$ with primary antibodies diluted in 
blocking solution. After washing with PBS, the cells were incubated for $1 \mathrm{~h}$ at room temperature with corresponding Cy3- or Alexa 488conjugated secondary antibodies diluted in blocking solution, washed again with PBS, and mounted in ProLong antifade reagent (Molecular Probes). We used blocking solution supplemented with $0.1 \%$ Triton X-100 instead of the standard blocking solution to permeabilize neurons to allow detection of intracellular molecules or the Myc epitope of expressed proteins. Living neurons were also immunostained with P84 $\mathrm{mAb}$ to SHPS- 1 by the use of the protocol described previously (Jareb and Banker, 1998). Fluorescence signals were observed with an AX-70 microscope (Olympus, Tokyo, Japan) and a $10 \times, 40 \times$, or $100 \times$ (numerical aperture, 1.35) objective. Digital images were captured with a cooled charge-coupled device camera (PXL; Photometrics, Tucson, AZ) and IPLab image analysis software (Scanalytics, Billerica, MA).

Quantitation of fluorescence signals. For quantitation of cell surface expression of SHPS-1, CD47, and mutants thereof, images were acquired with a $40 \times$ dry objective. Regions separated from the cell body by a distance of at least $80 \mu \mathrm{m}$ were chosen randomly for quantitative analysis of the localization of proteins in distal portions of neurites. Regions in which fasciculation was apparent were excluded from such analysis. One-pixel-wide linear segments (length, $\sim 50-100 \mu \mathrm{m}$ ) were traced along axons and dendrites. The average pixel intensity for each segment was determined and entered into Excel software (Microsoft, Redmond, WA) for data analysis. The average background intensity (based on neighboring regions of the culture that contained nontransfected cells) was subtracted from the values obtained for an axon or a dendrite, and an average axon/dendrite ratio was then calculated for each neuron. For each cDNA construct, 8-16 cells from at least two different culture preparations were examined.

To quantify the immunoreactivity for exogenous SHPS- 1 and CD47 at synaptic sites marked with GFP-VAMP-2 or GFP-actin, the ratio of presynaptic to adjacent axonal fluorescence signal or that of postsynaptic to adjacent dendritic fluorescence signal was determined for SHPS- 1 and CD47. The average pixel intensity at each GFP-VAMP-2-positive puncta was determined as an average presynaptic fluorescence intensity. To measure adjacent axonal fluorescence signal, 1-pixel-wide linear segments (length, 5-10 $\mu \mathrm{m}$ ) were traced along axons adjacent to the GFPVAMP-2-positive puncta, and the average pixel intensity for each segment was determined. To measure postsynaptic and adjacent dendritic fluorescence signal, the average pixel intensity for each GFP-actinpositive puncta and that for the adjacent dendritic region were determined, respectively. The average background intensity (based on neighboring regions of the culture that contained nontransfected cells) was subtracted from each value obtained, and an average presynapse/adjacent axon fluorescence ratio or an average postsynapse/adjacent dendrite fluorescence ratio was then calculated for each synapse. For each experiment, 19-26 synapses from at least two different culture preparations were examined.

\section{Results}

Differential distributions of endogenous SHPS-1 and CD47 in cultured hippocampal neurons

SHPS- 1 is virtually undetectable in the mouse embryonic nervous system, with the exception of the floor plate region of the spinal cord, where it is apparent as early as embryonic day 9; however, its expression gradually increases in the cerebellum and the retina during postnatal development (Chuang and Lagenaur, 1990; Mi et al., 2000). With the use of immunoblot analysis, we also found that the abundance of SHPS- 1 and CD47 in the rat hippocampus increases during postnatal development and that this temporal pattern of expression was recapitulated in cultured hippocampal neurons isolated from mouse embryos (see supplemental Fig. 1, available at www.jneurosci.org as supplemental material).

To examine the localization of SHPS- 1 and CD47 in neurons, we subjected mouse hippocampal neurons at 7 DIV to immunofluorescence analysis (Fig. 1). Immunoreactivity for SHPS-1 or
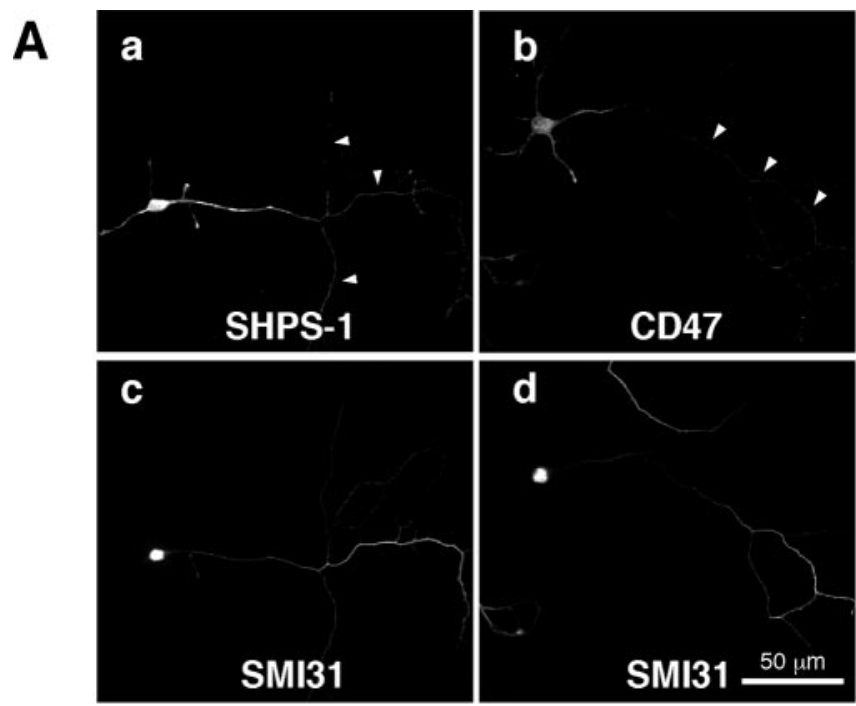

\section{B}
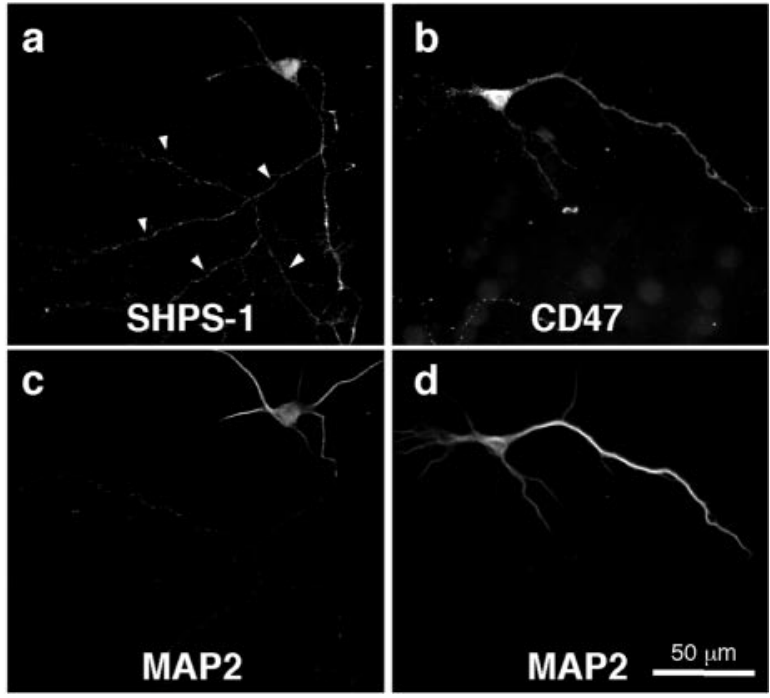

Figure 1. Localization of endogenous SHPS-1 and CD47 in mouse hippocampal neurons at 7 DIV. $\boldsymbol{A}$, Neurons were fixed and then stained both with a mAb to SHPS- 1 ( $\boldsymbol{a}$ ) or a mAb to CD47 (b) and with a mAb to phosphorylated neurofilaments (SMI31) $(\boldsymbol{c}, \boldsymbol{d}) . \boldsymbol{B}$, Neurons were fixed and then stained both with a mAb to SHPS-1 (a) or a mAb to CD47 (b) and with a mAb to MAP2 ( $\boldsymbol{c}$, $\boldsymbol{d})$. Arrowheads indicate SMI31-positive $(\boldsymbol{A})$ or MAP2-negative $(\boldsymbol{B})$ neurites. Scale bars, $50 \mu \mathrm{m}$. All results are representative of three separate experiments.

CD47 was detected at relatively low levels at this time, consistent with the immunoblot data. SHPS-1 immunoreactivity was present along axons, which were apparent as long, thin neurites with many branches that also stained with a mAb to phosphorylated neurofilaments, an axonal marker (Durham, 1990) (Fig. $1 A a, c)$. The SHPS-1-positive axons were not stained with a mAb to MAP2, a dendritic marker protein (Matus et al., 1981) (Fig. $1 \mathrm{Ba}, \mathrm{c})$. SHPS-1 immunoreactivity was also detected at the surface of the cell body and of dendrites, the latter of which were also positive for MAP2 (Fig. $1 \mathrm{Ba}, \mathrm{c}$ ). In contrast to SHPS-1, CD47 immunoreactivity was observed only at a low level along axons that were positive for phosphorylated neurofilaments (Fig. $1 A b, d)$. It was present at a higher level on the surface of MAP2positive dendrites and the cell body (Fig. $1 B b, d$ ). These results suggested that SHPS- 1 is localized to both axons and dendrites, whereas CD47 is primarily restricted to dendrites.

At 20 DIV, immunoreactivity for both SHPS-1 and CD47 in cultured mouse hippocampal neurons was markedly greater than 

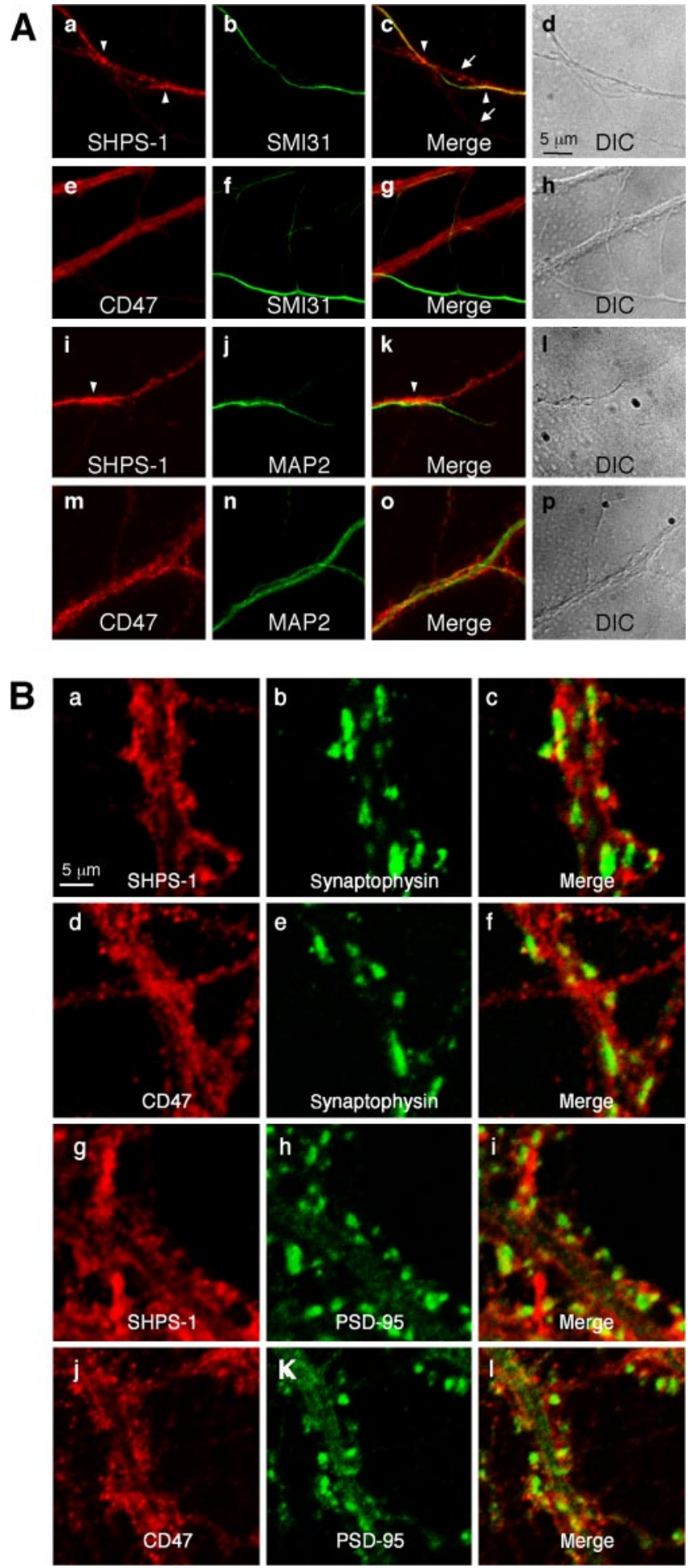

Figure 2. Localization of endogenous SHPS-1 and CD47 in mouse hippocampal neurons at 20 or 28 DIV. $A$, Cultured mouse hippocampal neurons were fixed at 20 DIV and then subjected to two-color immunofluorescence staining with the indicated pairs of mAbs. $\boldsymbol{c}, \boldsymbol{g}, \boldsymbol{k}$, and $\boldsymbol{o}$ are merged images of $\boldsymbol{a}$ and $\boldsymbol{b}, \boldsymbol{e}$ and $\boldsymbol{f}, \boldsymbol{i}$ and $\boldsymbol{j}$, and $\boldsymbol{m}$ and $\boldsymbol{n}$, respectively. Differential interference contrast (DIC) images corresponding to $\boldsymbol{a}-\boldsymbol{c}, \boldsymbol{e}-\boldsymbol{g}, \boldsymbol{i}-\boldsymbol{k}$, and $\boldsymbol{m}-\boldsymbol{o}$ are shown in $\boldsymbol{d}, \boldsymbol{h}, \boldsymbol{I}$, and $\boldsymbol{p}$ respectively. The arrows in cindicate SHPS- 1 immunoreactivity that was detected along SMI31negative neurites. Arrowheads in $\boldsymbol{a}$ and $\boldsymbol{c}$ indicate intense SHPS- 1 immunoreactivity at sites of contact between an SMI31-positive axon and the SMI31-negative dendrite-like neurite. The arrowheads in $\boldsymbol{i}$ and $\boldsymbol{k}$ indicate intense SHPS-1 immunoreactivity at sites of interaction between an SHPS-1-positive fiber and a MAP2-positive dendrite. $\boldsymbol{B}$, Neurons were fixed at 28 DIV and then subjected to two-color immunofluorescence staining with the indicated pairs of mAbs. $\boldsymbol{c}, \boldsymbol{f}$, $\boldsymbol{i}$, and $\boldsymbol{I}$ are merged images of $\boldsymbol{a}$ and $\boldsymbol{b}, \boldsymbol{d}$ and $\boldsymbol{e}, \boldsymbol{g}$ and $\boldsymbol{h}$, and $\boldsymbol{j}$ and $\boldsymbol{k}$, respectively. Scale bars, 5 $\mu \mathrm{m}$. All results are representative of three separate experiments. that apparent at 7 DIV (Fig. $2 A$ ). Individual neurons extended long axons and dendrites and formed multiple synapses with the neurites of other neurons, thereby generating a complex neuritic network. The localization of SHPS-1 and CD47 in single neurons was consequently difficult to determine. Nevertheless, we found that SHPS-1 immunoreactivity tended to be localized along SMI31-positive axons at this stage (Fig. $2 \mathrm{Aa}-d$ ). In contrast, the weak SHPS-1 immunoreactivity was observed along SMI31negative neurites (Fig. 2Ac, arrows) as well as MAP2-positive dendrites (Fig. $2 A i-l$ ). Immunoreactivity for CD47 was apparent along dendrites positive for MAP2 (Fig. $2 A m-p$ ); however, minimal CD47 immunoreactivity tended to be observed along SMI31-positive axons (Fig. $2 A e-h$ ). In addition, SHPS- 1 immunoreactivity was frequently concentrated at sites of contact between fibers positive for both SHPS-1 and SMI31 staining and dendrite-like neurites (Fig. $2 A a, c$, arrowheads). A similar concentration of SHPS-1 was also observed at contact sites between SHPS-1-positive fibers and MAP2-positive dendrites (Fig. 2Ai,k, arrowheads).

Both SHPS-1 and CD47 were shown previously to be present at ribbon synapses in the retina (Mi et al., 2000). We therefore next examined whether SHPS-1 or CD47 colocalized with presynaptic (synaptophysin) or postsynaptic (PSD-95) marker proteins in cultured mouse hippocampal neurons at $28 \mathrm{DIV}$. The distribution of SHPS-1 immunoreactivity appeared to be diffuse along neurites and overlapped in part with that of synaptophysin, which was detected in plaque-like structures corresponding to axonal synaptic termini on dendritic shafts (Fig. $2 \mathrm{Ba}-\mathrm{c}$ ). The SHPS-1 staining, however, was not colocalized with synaptophysin immunoreactivity. In addition, SHPS-1 immunoreactivity did not colocalize with that of PSD-95, which was detected in mushroom-like structures corresponding to spines on dendritic shafts (Fig. $2 \mathrm{Bg}-\mathrm{i}$ ). Furthermore, immunoreactivity for CD47 was apparent as dot-like staining along neurites but it did not colocalize with synaptophysin (Fig. $2 B d-f$ ) or PSD-95 (Fig. $2 B j-$ l). Accordingly, in contrast to the previous observation (Mi et al., 2000), these results suggested that SHPS-1 and CD47 are not specifically localized at synaptic sites in cultured mouse hippocampal neurons at 28 DIV.

\section{Differential localization of exogenously expressed SHPS-1 and CD47 in cultured hippocampal neurons}

We next examined the localization of recombinant SHPS-1 and CD47 in transfected hippocampal neurons. Cultured rat neurons were transfected with expression vectors for mouse SHPS-1 or mouse CD47, and the expression of these proteins was then detected by staining with mAbs to SHPS-1 (P84) or to CD47 (MIAP301). P84 and MIAP301 react specifically with mouse SHPS-1 and CD47, respectively, and do not cross-react with the corresponding rat proteins (data not shown). Exogenous SHPS-1 was distributed along long, thin axons as well as on dendrites and the cell body of nonpermeabilized neurons (Fig. 3Aa,b). In contrast, recombinant CD47 was localized predominantly to dendrites and the cell body, with only small amounts present along axons (Fig. 3Ac,d).

To determine the extent of axonal or dendritic localization of exogenous SHPS-1 or CD47, we cotransfected neurons with an expression vector for GFP, followed by fixation and staining with mAbs to SHPS-1 or to CD47 (Fig. 3B) under a nonpermeabilized condition. The ratio of axonal to dendritic fluorescence signals was then determined for each expressed protein. Although GFP appeared to be distributed diffusely throughout each cell, including all branches of the axon and dendrites (Fig. 3Bb,d), its axon/ 
A
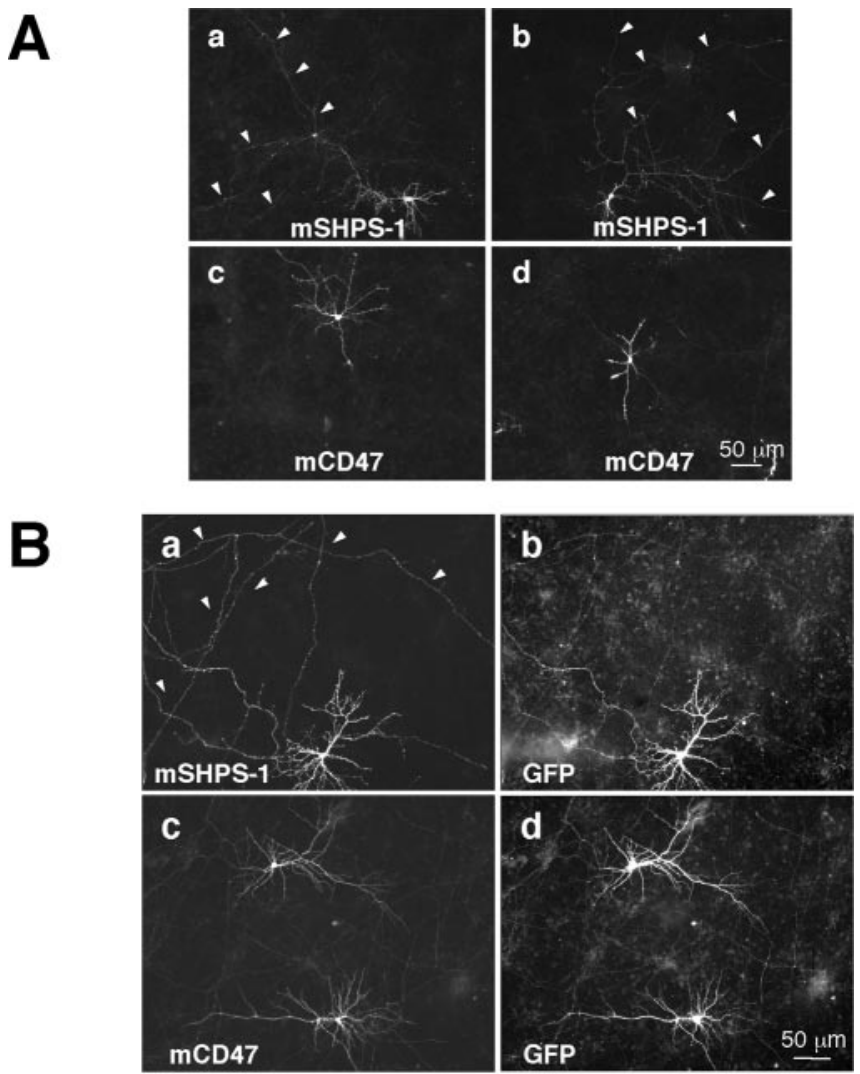

\section{C}

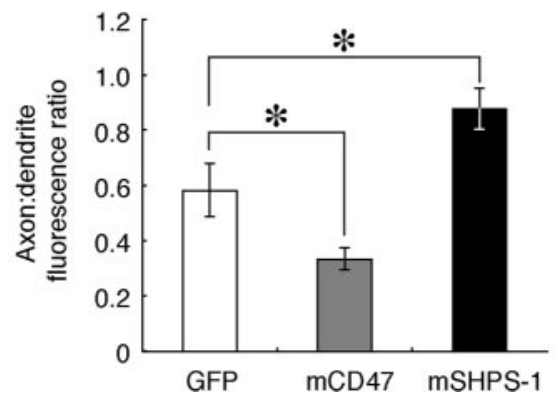

Figure 3. Localization of exogenously expressed mouse SHPS- 1 and mouse CD47 in cultured rat hippocampal neurons. $\boldsymbol{A}$, Neurons were transfected with expression vectors for mouse SHPS-1 (mSHPS-1) or mouse CD47 (mCD47), as indicated, at 12 DIV and fixed and immunostained with mAbs to mSHPS-1 or to mCD47, respectively, at 14 DIV. Arrowheads indicate long, thin fibers. $\boldsymbol{B}$, Neurons were cotransfected with expression vectors for GFP and either mSHPS-1 $(\boldsymbol{a}, \boldsymbol{b})$ or mCD47 $(\boldsymbol{c}, \boldsymbol{d})$ at 10 DIV and fixed and stained with mAbs to mSHPS-1 (a) or to mCD47 (c) at 14 DIV. Fluorescence signals of GFP are shown in $\boldsymbol{b}$ and $\boldsymbol{d}$. Arrowheads indicate long, thin fibers that were labeled intensely with the mAb to mSHPS-1. Scale bars, $50 \mu \mathrm{m}$. The results in $\boldsymbol{A}$ and $\boldsymbol{B}$ are representative of three separate experiments. $\boldsymbol{C}$, Quantitation of the distribution of exogenously expressed GFP, mCD47, and mSHPS-1 in transfected neurons. The axon/dendrite fluorescence ratio was calculated for each protein as described in Materials and Methods. Data are means \pm SE of values from 10 to 15 different cells. ${ }^{*} p<0.05$ for the indicated comparisons (Student's $t$ test).

dendrite fluorescence ratio was only $0.58 \pm 0.09$ (mean \pm SE; $n=$ 15) (Fig. 3C). Theoretically, one would expect a uniformly distributed protein, such as GFP, to have an axon/dendrite fluorescence ratio of 1 . However, the result above is possibly because GFP tended to accumulate in dendrites as a result of their relatively large diameter, as described previously (Cheng et al., 2002). The axon/dendrite fluorescence ratio for SHPS-1 $(0.88 \pm 0.07$; $n=10 ; p<0.05)$ was markedly greater than that for GFP, whereas that for $\operatorname{CD} 47(0.33 \pm 0.04 ; n=10 ; p<0.05)$ was
A
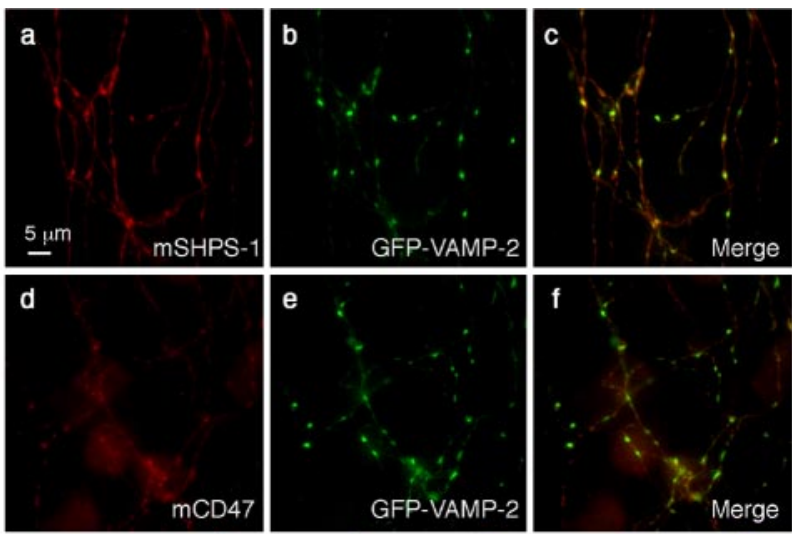

B
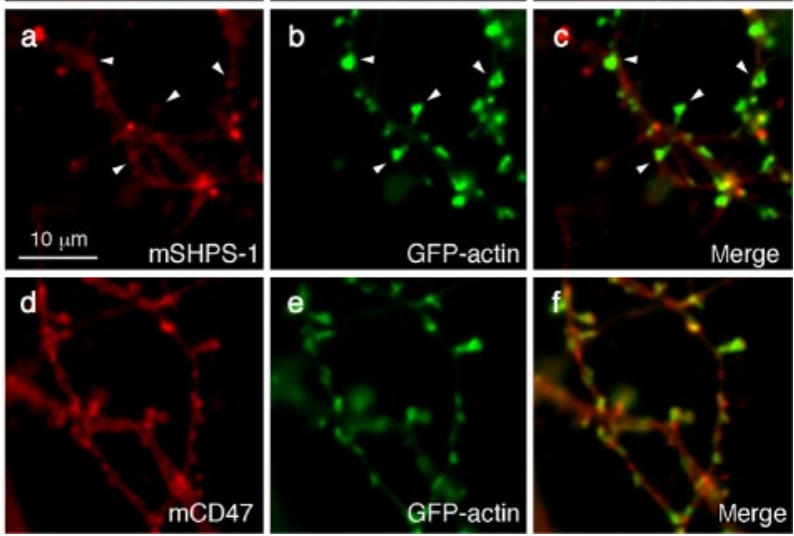

Figure 4. Localization of exogenously expressed SHPS-1 or CD47 relative to that of synaptic marker proteins in cultured rat hippocampal neurons. Neurons were cotransfected with expression vectors for GFP-VAMP-2 ( $\boldsymbol{A}$ ) or GFP-actin ( $\boldsymbol{B})$ and either mouse SHPS-1 (mSHPS-1) ( $\boldsymbol{a}-\boldsymbol{c})$ or mCD47 (d-f) at 14 DIV. Seven days after transfection, the neurons were fixed and stained with mAbs to mSHPS-1 $(\boldsymbol{a})$ or to $\mathrm{mCD} 47(\boldsymbol{d})$. The fluorescence signals of GFP-VAMP-2 $(\boldsymbol{A})$ or GFP-actin $(\boldsymbol{B})$ are shown in $\boldsymbol{b}$ and $\boldsymbol{e}$. Merged images are shown in $\boldsymbol{c}$ and $\boldsymbol{f}$. Arrowheads in $\boldsymbol{B a}-\boldsymbol{c}$ indicate intense CD47 immunoreactivity at dendritic spine sites marked with GFP-actin. Scale bars: $A, 5 \mu \mathrm{m} ; \boldsymbol{B}, 10 \mu \mathrm{m}$. All results are representative of three separate experiments.

substantially smaller than that for GFP (Fig. 3C). We also stained living neurons with $\mathrm{mAb}$ to SHPS- 1 before the fixation. In such an experiment, the axon/dendrite fluorescence ratio for SHPS-1 $(0.99 \pm 0.12 ; n=8)$ was comparable with that apparent with neurons fixed under a nonpermeabilized condition. The present results above thus suggested that exogenously expressed SHPS-1 was preferentially localized to axons compared with GFP and CD47, whereas exogenously expressed CD47 was preferentially localized to dendrites, although the extent of the polarization of SHPS-1 or CD47 was weaker than that of polarized molecules as reported previously (Cheng et al., 2002; Rivera et al., 2003; Sampo et al., 2003).

We also compared the localization of recombinant SHPS-1 with that of GFP-tagged VAMP-2 (GFP-VAMP-2), which accumulates at presynaptic sites (Ahmari et al., 2000). Cotransfection of neurons with vectors for SHPS- 1 and GFP-VAMP-2 revealed the presence of SHPS-1 along GFP-VAMP-2-positive neurites as well as its colocalization with GFP-VAMP-2 at presynaptic sites $($ Fig. $4 A a-c)$. In contrast, cotransfection of neurons with vectors for CD47 and GFP-VAMP-2 revealed that CD47 immunoreactivity was minimal along GFP-VAMP-2-positive neurites and was not colocalized with GFP-VAMP-2 at presynaptic sites (Fig. $4 A d-f$ ). The ratio of presynaptic to adjacent axonal fluorescence signals at each synapse was determined for SHPS- 1 and CD47. The presynapse/adjacent axon fluorescence ratio for exogenous 
A
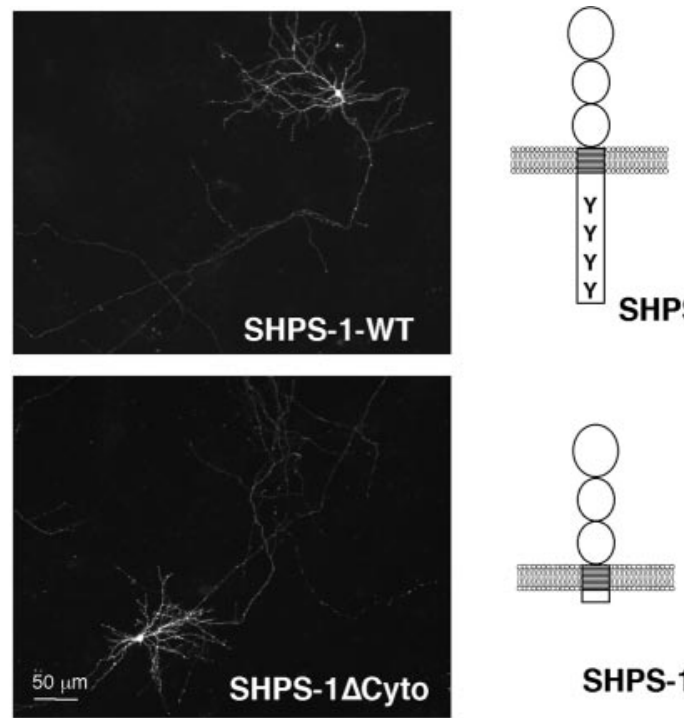

SHPS- $1 \Delta$ Cyto

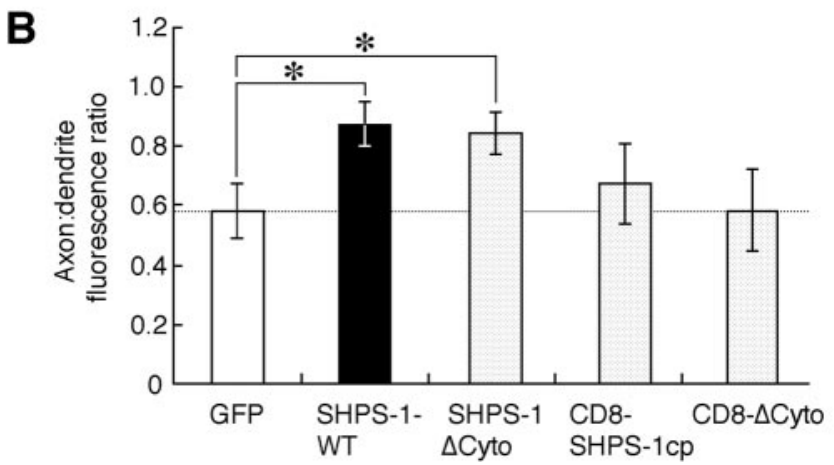

Figure 5. Localization of wild-type SHPS-1 and the mutant SHPS-1 $\Delta$ Cyto in cultured rat hippocampal neurons. $\boldsymbol{A}$, Neurons were transfected with an expression vector for wild-type mouse SHPS-1 (mSHPS-1) (SHPS-1-WT) or for SHPS-1 $\Delta$ Cyto at 12 DIV. The neurons were fixed at 14 DIV and immunostained with a mAb to mSHPS-1. Schematic structures of the wild-type and mutant SHPS-1 proteins are shown to the right of each fluorescence image. Scale bar, 50 $\mu \mathrm{m}$. Results are representative of three separate experiments. $\boldsymbol{B}$, Quantitation of the axon/ dendrite fluorescence ratio for exogenously expressed GFP, wild-type mSHPS-1, SHPS-1 $\Delta$ Cyto, CD8-SHPS-1cp, or CD8- $\Delta$ Cyto in cultured rat hippocampal neurons. Expression of the CD8 chimeric proteins was detected with a mAb to human CD8. Data are means \pm SE of values from 8 to 15 different cells. ${ }^{*} p<0.05$ for the indicated comparisons (Student's $t$ test).

SHPS-1 $(2.72 \pm 0.25 ; n=22)$ was markedly greater than that for exogenous CD47 (1.76 $\pm 0.18 ; n=19 ; p<0.01)$. We also compared the distribution of exogenous SHPS-1 with that of a GFPactin fusion protein that accumulates in dendritic spines (Fischer et al., 1998). Recombinant SHPS-1 did not colocalize with GFPactin at dendritic spines (Fig. $4 B a-c$ ). In contrast, recombinant CD47 was frequently colocalized with GFP-actin at these structures (Fig. $4 B d-f$ ). The postsynapse/adjacent dendrite fluorescence ratio for exogenous CD47 (2.06 $\pm 0.27 ; n=20)$ was markedly greater than that for exogenous SHPS-1 $(1.45 \pm 0.14 ; n=26$; $p<0.05)$.

Determination of the domains of SHPS-1 and CD47 required for their localization

To determine which regions of SHPS-1 and CD47 are required for their specific localization in cultured neurons, we first compared the distribution of the SHPS-1 mutant SHPS-1DCyto (which comprises amino acids 1-404 of mouse SHPS-1 and
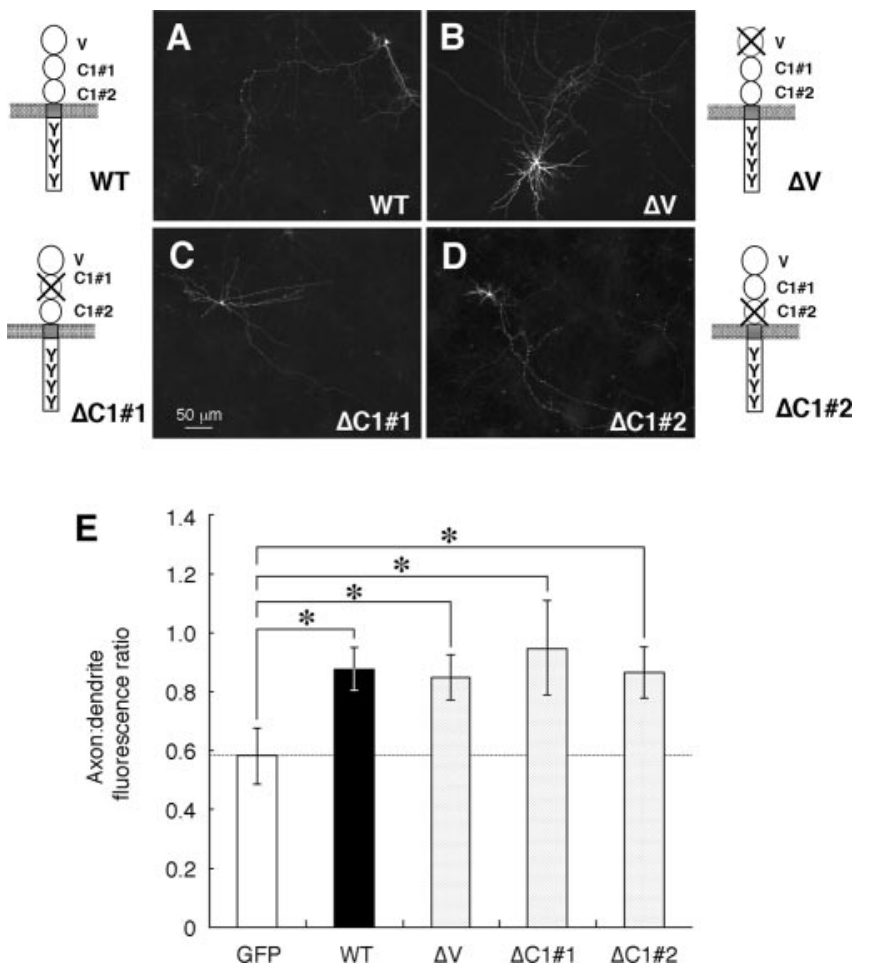

Figure 6. Localization of the SHPS-1 mutants $\Delta \mathrm{V}, \Delta C 1 \# 1$, and $\Delta C 1 \# 2$ in cultured rat hippocampal neurons. $\boldsymbol{A}-\boldsymbol{D}$, Neurons were transfected with expression vectors for wild-type (WT) mouse SHPS-1 (mSHPS-1) (A) or for the SHPS-1 mutants $\Delta V(B), \Delta C \# 1(C)$, or $\Delta C 1 \# 2$ (D) at 12 DIV. They were fixed at 14 DIV and immunostained with P84 $(\boldsymbol{A}, \boldsymbol{B}, \boldsymbol{D})$ or FG2 $(\boldsymbol{C}) \mathrm{mAbs}$ to SHPS-1. Schematic structures of the wild-type and mutant proteins are shown at the side of each image. Scale bar, $50 \mu \mathrm{m}$. Results are representative of three separate experiments. $\boldsymbol{E}$, Quantitation of the axon/dendrite fluorescence ratio for exogenously expressed GFP, wild-type mSHPS-1, or the SHPS-1 mutants $\Delta V, \Delta C 1 \# 1$, or $\Delta C 1 \# 2$. Data are means \pm SE of values from 8 to 16 different cells. ${ }^{*} p<0.05$ for the indicated comparisons (Student's $t$ test).

therefore lacks most of the cytoplasmic portion of the intact protein) with that of wild-type SHPS-1 in transfected rat hippocampal neurons. SHPS-1 $\Delta$ Cyto was detected along axons, and its localization was virtually indistinguishable from that of wild-type SHPS-1 (Fig. 5A). The axon/dendrite fluorescence ratio for SHPS- $1 \Delta$ Cyto was $0.84 \pm 0.07(n=11)$ (Fig. $5 B)$, suggesting that the cytoplasmic portion of mouse SHPS-1 is not required for its predominantly axonal localization. We next generated expression vectors both for a chimeric protein comprising the extracellular and transmembrane regions of human CD8 fused to the cytoplasmic region of mouse SHPS-1 (CD8-SHPS-1cp) and for a chimeric protein, CD8- $\Delta$ Cyto, that lacks the cytoplasmic region of CD8-SHPS-1cp. The axon/dendrite fluorescence ratios for CD8-SHPS- $1 \mathrm{cp}(0.68 \pm 0.13 ; n=8)$ and for CD8- $\Delta$ Cyto $(0.58 \pm$ $0.14 ; n=8)$ were similar to that of GFP ( $p=0.57$ or 0.99 , respectively) (Fig. $5 B$ ). These results therefore suggested that the extracellular region of SHPS-1 is required for its preferential localization to axons.

SHPS-1 possesses three Ig-like domains in its extracellular region (Fujioka et al., 1996; Sano et al., 1997); the first is homologous to a V-type Ig domain, whereas the second and third resemble a C1-type Ig domain (Williams and Barclay, 1988). We next examined which of these three domains is required for the axonal localization of SHPS-1. We generated three cDNAs encoding SHPS-1 mutants designated $\Delta \mathrm{V}, \Delta \mathrm{C} 1 \# 1$, and $\Delta \mathrm{C} 1 \# 2$, which lack the first $\left(\mathrm{NH}_{2}\right.$-terminal), second, and third Ig domains, respectively. Rat hippocampal neurons were transfected 
with expression vectors for these mutants, and the distribution of each mutant was compared with that of wild-type SHPS-1. To detect the expression of $\Delta \mathrm{C} 1 \# 1$, we used the mAb FG2 instead of P84, which reacts with the second Ig domain of SHPS- 1 and thus failed to recognize $\Delta \mathrm{C} 1 \# 1$ (data not shown). The axonal localization of $\Delta \mathrm{V}, \Delta \mathrm{C} 1 \# 1$, or $\Delta \mathrm{C} 1 \# 2$ appeared identical to that of wildtype SHPS-1 (Fig. 6A-D). The axon/dendrite fluorescence ratio for each mutant $[0.85 \pm 0.08$ for $\Delta \mathrm{V}(n=16), 0.95 \pm 0.16$ for $\Delta \mathrm{C} 1 \# 1$ $(n=8), 0.87 \pm 0.09$ for $\Delta \mathrm{C} 1 \# 2(n=10)]$ was therefore similar to that for the wild-type protein and substantially greater than that for GFP $(p<0.05)$ (Fig. $6 E$ ). These results suggested that the predominant axonal localization of SHPS- 1 is not mediated by any single Ig domain in the extracellular region of this protein.

CD47 possesses a V-type Ig-like extracellular region, five putative transmembrane segments, and a short cytoplasmic tail (Brown and Frazier, 2001). We next examined which regions of CD47 are required for its dendritic localization by transfecting cultured rat hippocampal neurons with expression vectors for a mutant of mouse CD47 that lacks the short cytoplasmic tail at the $\mathrm{COOH}$ terminus (CD47 $\Delta$ Cterm) or for a chimeric protein comprising the extracellular Ig region of human CD8 fused to the multiple membrane-spanning domains and short cytoplasmic tail of mouse CD47 (CD8-CD47MMS). The CD47 $\Delta$ Cterm mutant localized predominantly to dendrites in a pattern almost identical to that of wild-type CD47 (Fig. 7A,B). The axon/dendrite fluorescence ratio for CD47 $\Delta$ Cterm $(0.44 \pm 0.05 ; n=11)$ was thus similar to that for wild-type CD47 ( $p=0.13$ ) (Fig. $7 D)$. In contrast, CD8-CD47MMS appeared to localize to both axons and dendrites (Fig. $7 C$ ); the axon/dendrite fluorescence ratio $(0.71 \pm 0.08 ; n=8)$ was thus similar to that of GFP $(p=0.40)$. These results suggested that the extracellular region of CD47 is necessary for its dendritic localization.

Accumulation of SHPS-1 and CD47 at neuritic contact sites of transfected hippocampal neurons

To investigate the functional consequences of the interaction between SHPS-1 (on axons) and CD47 (on dendrites), we first transfected rat hippocampal neurons independently with expression vectors for mouse SHPS-1 and Myc epitope-tagged mouse CD47. Two-color immunofluorescence staining revealed that SHPS- 1 and CD47myc were expressed predominantly along axons and dendrites, respectively, and that both proteins accumulated at sites of contact between axons and dendrites (Fig. 8A). The average number of accumulations per a single CD47expressing neuron was $9.3 \pm 3.2(n=4)$ when CD47-expressing neurons were cultured with wild-type SHPS-1-expressing neurons. Such accumulation of CD47myc was not observed when neurons were transfected with an expression vector for CD8$\Delta$ Cyto instead of that for wild-type SHPS-1 (Fig. $8 B$ ), suggesting that accumulation and colocalization of SHPS-1 and CD47 at neuritic contact sites are mediated by specific interaction of the extracellular region of SHPS-1 with that of CD47. This notion received additional support on examination of neurons transfected with expression vectors for CD47 and for the $\Delta V$ mutant of SHPS-1 tagged with the Myc epitope. SHPS-1 interacts with the extracellular region of CD47 through its $\mathrm{NH}_{2}$-terminal V-type Ig domain (Vernon-Wilson et al., 2000). The $\Delta \mathrm{V}$ mutant of SHPS-1 and wild-type CD47 did not accumulate at sites of contact between axons and dendrites (Fig. 8C). The accumulation of SHPS-1 and CD47 may not be attributable to blebbing and dying neurons, because such accumulation was only observed at sites of contact between SHPS-1-expressing neurons and CD47expressing neurons. At higher magnification, the sites of contact
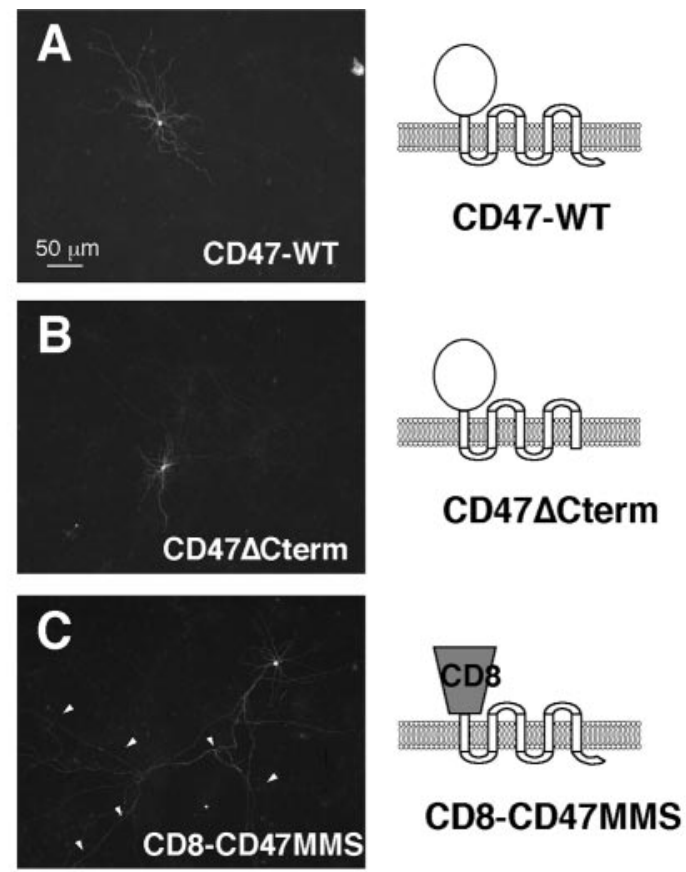

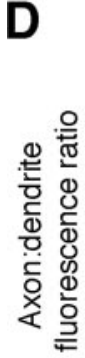

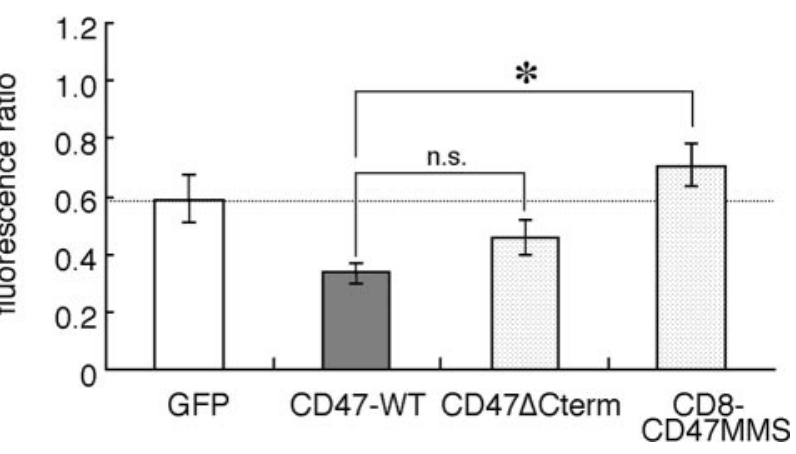

Figure 7. Localization of wild-type and mutant CD47 proteins in cultured rat hippocampal neurons. $A-C$, Neurons were transfected with expression vectors for wild-type mCD47 (CD47WT) $(\boldsymbol{A})$, the mCD47 mutant CD47 $\Delta$ Cterm $(\boldsymbol{B})$, or the chimeric protein $\mathrm{CD} 8$-CD47MMS $(\boldsymbol{C})$ at 12 DIV. They were fixed and immunostained with mAbs to $\mathrm{mCD} 47(\boldsymbol{A}, \boldsymbol{B})$ or to human $\mathrm{CD8}(\boldsymbol{C})$ at 14 DIV. Scale bar, $50 \mu \mathrm{m}$. Data are representative of three separate experiments. $D$, Quantitation of the axon/dendrite fluorescence ratio for exogenously expressed GFP, wild-type mCD47, CD47 $\Delta$ Cterm, or CD8-CD47MMS. Data are means \pm SE of values from 8 to 15 different cells. ${ }^{*} p<0.05$ for the indicated comparison (Student's $t$ test). n.s., Not significant.

between axons with accumulated wild-type SHPS-1 and dendrites with accumulated wild-type CD47 appeared as enlarged, dot-like structures (Fig. 8Da,b). Immunoreactivity for endogenous VAMP-2 was not detected in association with these structures (Fig. $8 D c$ ), suggesting that they are distinct from mature synapses.

\section{Discussion}

We have now shown that endogenous SHPS-1 and CD47 are localized differentially on the surface of cultured hippocampal neurons, with SHPS-1 being present on both axons and dendrites and CD47 being essentially restricted to dendrites. A dendritic localization was also apparent for recombinant CD47 expressed in hippocampal neurons, whereas recombinant SHPS-1 was localized preferentially to axons rather than to dendrites. An axonal 
A
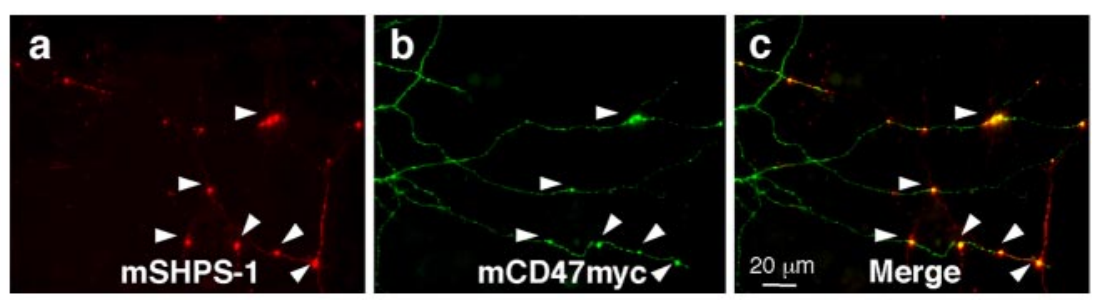

B
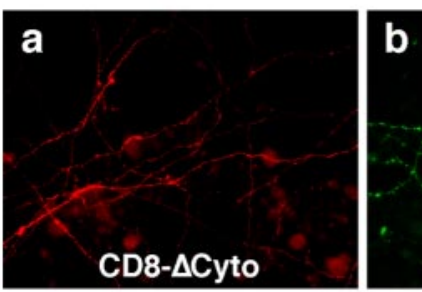

C
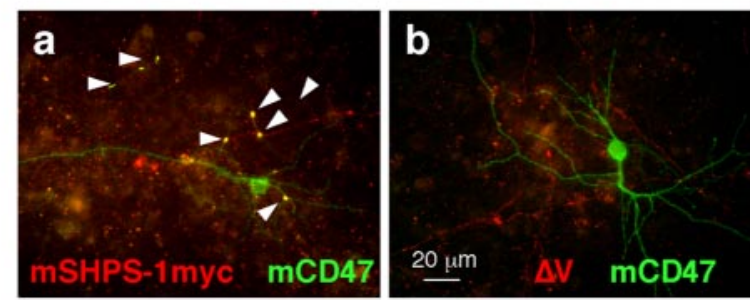
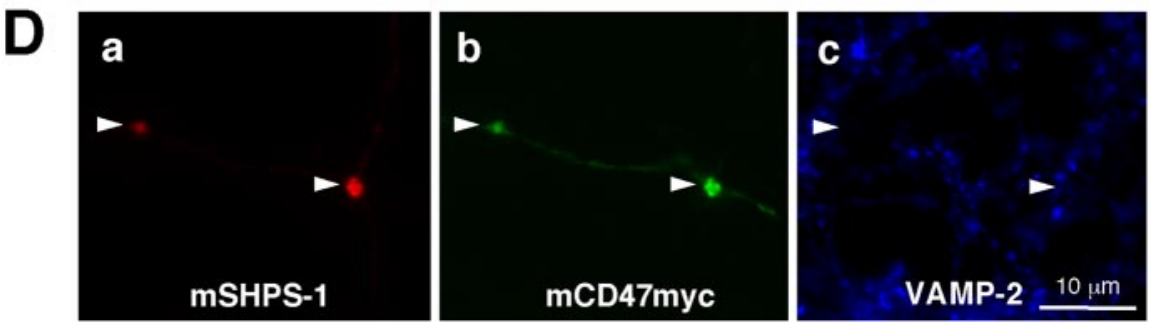

Figure 8. Accumulation of exogenously expressed SHPS-1 and CD47 at sites of contact between neurites of cultured rat hippocampal neurons. $A, B$, Neurons were transfected first with an expression vector for mCD47myc at 12 DIV and then $24 \mathrm{~h}$ later with an expression vector for mouse SHPS-1 (mSHPS-1) $(\boldsymbol{A})$ or for CD8- $\Delta$ Cyto $(\boldsymbol{B})$. Seven days after the second transfection, neurons were fixed and subjected to two-color immunostaining with mAbs to mSHPS-1 ( $\mathbf{A a})$ or to human CD8 (Ba) and with a $\mathrm{mAb}$ to the Myc epitope $(\boldsymbol{b})$. Merged images are shown in $\boldsymbol{c}$. Scale bars, $20 \mu \mathrm{m}$. $\boldsymbol{C}$, Neurons were transfected first with an expression vector for mCD47 at 12 DIV and then $24 \mathrm{~h}$ later with an expression vector for mSHPS-1myc (a) or for the Myc epitopetagged $\Delta V$ mutant of mSHPS-1 $(\boldsymbol{b})$. Seven days after the second transfection, neurons were fixed and subjected to two-color immunostaining with mAbs to the Myc epitope (red) and to mCD47 (green). Scale bar, $20 \mu \mathrm{m}$. D, Neurons were transfected with expression vectors for wild-type mSHPS-1 and mCD47myc and then fixed as in $\boldsymbol{A}$. They were then subjected to three-color immunostaining with a mAb to mSHPS-1 (a), a mAb to the Myc epitope ( $\boldsymbol{b}$ ), and polyclonal antibodies to VAMP-2 (c). Scale bar, 10 $\mu \mathrm{m}$. All arrowheads indicate the accumulation of SHPS- 1 and CD47 at sites of contact between neuronal processes.

localization for SHPS-1 was suggested previously on the basis of an immunohistochemical study of the cerebellum (Comu et al., 1997). In addition, glycoprotein gp93, which had been previously purified biochemically from growth cone membranes, was recently shown to be identical to SHPS-1 (Wang et al., 2003). Our present results thus suggest that SHPS-1 and CD47, as a result of their differential distribution on axons and dendrites, respectively, constitute a directional intercellular communication system in the CNS.

Forced expression of SHPS-1 and CD47 in cultured hippocampal neurons revealed that the former accumulated with GFP-VAMP-2 at presynaptic sites and the latter accumulated with GFP-actin at postsynaptic sites. These results are consistent with the previous observation that both SHPS-1 and CD47 are present at ribbon synapses in the retina (Mi et al., 2000). How- ever, we also found that endogenous SHPS- 1 and CD47 did not colocalize precisely with synaptic marker proteins but rather were localized diffusely along neurites, including perisynaptic sites, at 28 DIV. The neural adhesion molecules $\mathrm{N}$-cadherin and nectin have been found to localize to perisynaptic sites, such as puncta adherentia junctions, at the late stage of synaptic maturation, although these proteins were localized to synaptic sites at early stages (Mizoguchi et al., 2002). Furthermore, N-cadherin present at synaptic sites was dispersed laterally along the plasma membrane in response to depolarization by high $\mathrm{K}^{+}$in cultured hippocampal neurons (Tanaka et al., 2000). The localization of SHPS-1 or CD47 might thus also be regulated in a manner dependent of neuronal development or synaptic activity.

We have shown that the extracellular region of SHPS-1 is required for its axonal localization; however, the localization of SHPS- 1 to axons was not mediated by any single Ig domain in the extracellular region of the protein. Although the mechanisms underlying the axonal localization of membrane proteins are not well understood, both selective delivery and selective retention have been proposed to play a role (Burack et al., 2000). The distribution of the neural adhesion molecule NgCAM, which is primarily restricted to the axonal surface (Jareb and Banker, 1998; Silverman et al., 2001), requires the extracellular region of the protein (Sampo et al., 2003). In addition, the extracellular region of $\mathrm{Ng}$ CAM is implicated as a targeting signal that mediates sorting of the protein into axonal transport carriers that preferentially deliver their cargo proteins to the axonal membrane (Sampo et al., 2003). More than one of the five fibronectin type III-like domains in the extracellular region of NgCAM is required for function of the axonal localization signal of this protein (Sampo et al., 2003), an observation similar to the situation for SHPS-1 demonstrated here. The extracellular region of SHPS-1, like that of NgCAM, might thus also mediate the sorting of SHPS-1 into axonal transport carriers. $\mathrm{N}$-glycosylation has been suggested to mediate, in part, the axonal sorting of amyloid precursor protein (Tienari et al., 1996). Given that SHPS-1 is highly $N$-glycosylated (Sano et al., 1997; Yamao et al., 1997), its $N$-glycosylation also might contribute to the sorting of this protein into axonal transport carriers.

Two types of sorting signal, tyrosine-based motifs (Jareb and Banker, 1998) and dileucine-based motifs (Poyatos et al., 2000; Rivera et al., 2003), present in the cytoplasmic regions of membrane proteins have been identified as mediators of dendritic localization. However, our results indicate that the extracellular region of CD47, rather than its cytoplasmic region, is necessary 
for the dendritic localization of this protein. CD47 associates through its extracellular region with integrins, such as $\alpha \operatorname{IIb} \beta 3$, $\alpha \mathrm{v} \beta 3$, and $\alpha 2 \beta 1$, and regulates integrin function (Brown and Frazier, 2001). Various integrin subunits, including $\alpha \mathrm{v}, \alpha 2, \beta 1$, and $\beta 3$, are expressed in the hippocampus (Chan et al., 2003), although their precise localization in hippocampal neurons has not been determined. The integrin $\alpha 5$ subunit, however, which couples with the $\beta 1$ subunit, has been found to localize to apical dendrites of pyramidal cells in the hippocampus (Bi et al., 2001). The dendritic localization of integrins that associate with CD47 might therefore determine the localization of the latter protein in hippocampal neurons.

Endogenous SHPS-1 was found frequently to be enriched at contact sites between axons and dendrites. Forced expression of either SHPS-1 or CD47 in independent neurons also resulted in marked accumulation of both proteins at sites of neurite contact. The SHPS- 1 mutant $\Delta \mathrm{V}$, which lacks the $\mathrm{NH}_{2}$-terminal V-type Ig domain, failed to generate such accumulation. The sites of contact between SHPS-1-positive axons and CD47-positive dendrites did not contain the synaptic marker protein VAMP-2, however, suggesting that SHPS-1 and CD47 might function as neuronal adhesion molecules.

The physiological functions of the CD47-SHPS-1 system in the CNS remain to be fully elucidated. However, we recently showed that CD47, through its interaction with SHPS-1, promotes neurite and filopodium formation in N1E-115 neuroblastoma cells in a manner dependent on Rac and Cdc42 (Miyashita et al., 2004). Furthermore, whereas expression of CD47 alone increased the number of dendrites emanating from the cell body, the interaction of CD47 with SHPS-1 was required for promotion of filopodium formation in cultured hippocampal neurons (Miyashita et al., 2004; H. Ohnishi, T. Murata, and T. Matozaki, unpublished observations). During the early stage of dendritic spine formation, filopodia emerge from dendrites at sites of contact with an axon. These filopodia subsequently form nascent synapses at the contact sites and develop bulbous heads, eventually growing into mature spines as synaptogenesis proceeds (Fiala et al., 2002). On the basis of our present results, we now suggest that CD47, which is localized predominantly to dendrites, promotes the formation of filopodia on dendrites through its interaction with SHPS-1 expressed on a neighboring axon. The activation of Rac and Cdc42 and consequent reorganization of the actin cytoskeleton induced by the interaction of dendritic CD47 with axonal SHPS-1 might then further contribute to formation of mature dendritic spines. It remains to be determined whether the enlarged structure observed at sites of contact between neurites expressing SHPS- 1 and those expressing CD47 in the present study represent the bulbous heads of immature spines.

\section{References}

Ahmari SE, Buchanan J, Smith SJ (2000) Assembly of presynaptic active zones from cytoplasmic transport packets. Nat Neurosci 3:445-451.

Araki T, Yamada M, Ohnishi H, Sano S, Hatanaka H (2000) BIT/SHPS-1 enhances brain-derived neurotrophic factor-promoted neuronal survival in cultured cerebral cortical neurons. J Neurochem 75:1502-1510.

Bi X, Lynch G, Zhou J, Gall CM (2001) Polarized distribution of $\alpha 5$ integrin in dendrites of hippocampal and cortical neurons. J Comp Neurol 435:184-193.

Brown EJ, Frazier WA (2001) Integrin-associated protein (CD47) and its ligands. Trends Cell Biol 11:130-135.

Burack MA, Silverman MA, Banker G (2000) The role of selective transport in neuronal protein sorting. Neuron 26:465-472.

Chan CS, Weeber EJ, Kurup S, Sweatt JD, Davis RL (2003) Integrin requirement for hippocampal synaptic plasticity and spatial memory. J Neurosci 23:7107-7116.
Cheng C, Glover G, Banker G, Amara SG (2002) A novel sorting motif in the glutamate transporter excitatory amino acid transporter 3 directs its targeting in Madin-Darby canine kidney cells and hippocampal neurons. J Neurosci 22:10643-10652.

Chuang W, Lagenaur CF (1990) Central nervous system antigen P84 can serve as a substrate for neurite outgrowth. Dev Biol 137:219-232.

Comu S, Weng W, Olinsky S, Ishwad P, Mi Z, Hempel J, Watkins S, Lagenaur CF, Narayanan V (1997) The murine P84 neural adhesion molecule is SHPS-1, a member of the phosphatase-binding protein family. J Neurosci 17:8702-8710.

Durham HD (1990) Demonstration of hyperphosphorylated neurofilaments in neuronal perikarya in vivo by microinjection of antibodies into cultured spinal neurons. J Neuropathol Exp Neurol 49:582-590.

El Far O, Charvin N, Leveque C, Martin-Moutot N, Takahashi M, Seagar MJ (1995) Interaction of a synaptobrevin (VAMP)-syntaxin complex with presynaptic calcium channels. FEBS Lett 361:101-105.

Fiala JC, Allwardt B, Harris KM (2002) Dendritic spines do not split during hippocampal LTP or maturation. Nat Neurosci 5:297-298.

Fields RD, Stevens-Graham B (2002) New insight into neuron-glia communication. Science 298:556-562.

Fischer M, Kaech S, Knutti D, Matus A (1998) Rapid actin-based plasticity in dendritic spines. Neuron 20:847-854.

Fujioka Y, Matozaki T, Noguchi T, Iwamatsu A, Yamao T, Takahashi N, Tsuda M, Takada T, Kasuga M (1996) A novel membrane glycoprotein, SHPS-1, that binds the SH2-domain-containing protein tyrosine phosphatase SHP-2 in response to mitogens and cell adhesion. Mol Cell Biol 16:6887-6899.

Hayashi K, Kawai-Hirai R, Ishikawa K, Takata K (2002) Reversal of neuronal polarity characterized by conversion of dendrites into axons in neonatal rat cortical neurons in vitro. Neuroscience 110:7-17.

Jareb M, Banker G (1998) The polarized sorting of membrane proteins expressed in cultured hippocampal neurons using viral vectors. Neuron 20:855-867.

Jiang P, Lagenaur CF, Narayanan V (1999) Integrin-associated protein is a ligand for the P84 neural adhesion molecule. J Biol Chem 274:559-562.

Kamiguchi H, Lemmon V (2000) IgCAMs: bidirectional signals underlying neurite growth. Curr Opin Cell Biol 12:598-605.

Kharitonenkov A, Chen Z, Sures I, Wang H, Schilling J, Ullrich A (1997) A family of proteins that inhibit signalling through tyrosine kinase receptors. Nature 386:181-186.

Matus A, Bernhardt R, Hugh-Jones T (1981) High molecular weight microtubule-associated proteins are preferentially associated with dendritic microtubules in brain. Proc Natl Acad Sci USA 78:3010-3014.

Mi ZP, Jiang P, Weng WL, Lindberg FP, Narayanan V, Lagenaur CF (2000) Expression of a synapse-associated membrane protein, P84/SHPS-1, and its ligand, IAP/CD47, in mouse retina. J Comp Neurol 416:335-344.

Miyashita M, Ohnishi H, Okazawa H, Tomonaga H, Hayashi A, Fujimoto TT, Furuya N, Matozaki T (2004) Promotion of neurite and filopodium formation by CD47: roles of integrins, Rac, and Cdc42. Mol Biol Cell 15:3950-3963.

Mizoguchi A, Nakanishi H, Kimura K, Matsubara K, Ozaki-Kuroda K, Katata T, Honda T, Kiyohara Y, Heo K, Higashi M, Tsutsumi T, Sonoda S, Ide C, Takai Y (2002) Nectin: an adhesion molecule involved in formation of synapses. J Cell Biol 156:555-565.

Motegi S, Okazawa H, Ohnishi H, Sato R, Kaneko Y, Kobayashi H, Tomizawa K, Ito T, Honma N, Bühring HJ, Ishikawa O, Matozaki T (2003) Role of the CD47-SHPS-1 system in regulation of cell migration. EMBO J 22:2634-2644.

Murase S, Schuman EM (1999) The role of cell adhesion molecules in synaptic plasticity and memory. Curr Opin Cell Biol 11:549-553.

Niwa H, Yamamura K, Miyazaki J (1991) Efficient selection for highexpression transfectant with a novel eukaryotic vector. Gene 108:193-199.

Ohnishi H, Kubota M, Ohtake A, Sato K, Sano S (1996) Activation of protein-tyrosine phosphatase SH-PTP2 by a tyrosine-based activation motif of a novel brain molecule. J Biol Chem 271:25569-25574.

Ohnishi H, Yamada M, Kubota M, Hatanaka H, Sano S (1999) Tyrosine phosphorylation and association of BIT with SHP-2 induced by neurotrophins. J Neurochem 72:1402-1408.

Oldenborg PA, Zheleznyak A, FangYF, Lagenaur CF, Gresham HD, Lindberg FP (2000) Role of CD47 as a marker of self on red blood cells. Science 288:2051-2054. 
Poyatos I, Ruberti F, Martinez-Maza R, Gimenez C, Dotti CG, Zafra F (2000) Polarized distribution of glycine transporter isoforms in epithelial and neuronal cells. Mol Cell Neurosci 15:99-111.

Reinhold MI, Lindberg FP, Plas D, Reynolds S, Peters MG, Brown EJ (1995) In vivo expression of alternatively spliced forms of integrin-associated protein (CD47). J Cell Sci 108:3419-3425.

Rivera JF, Ahmad S, Quick MW, Liman ER, Arnold DB (2003) An evolutionarily conserved dileucine motif in Shal $\mathrm{K}^{+}$channels mediates dendritic targeting. Nat Neurosci 6:243-250.

Sampo B, Kaech S, Kunz S, Banker G (2003) Two distinct mechanisms target membrane proteins to the axonal surface. Neuron 37:611-624.

Sano S, Ohnishi H, Omori A, Hasegawa J, Kubota M (1997) BIT, an immune antigen receptor-like molecule in the brain. FEBS Lett 411:327-334

Sato R, Ohnishi H, Kobayashi H, Kiuchi D, Hayashi A, Kaneko Y, Honma N, Okazawa H, Hirata Y, Matozaki T (2003) Regulation of multiple functions of SHPS-1, a transmembrane glycoprotein, by its cytoplasmic region. Biochem Biophys Res Commun 309:584-590.

Seiffert M, Cant C, Chen Z, Rappold I, Brugger W, Kanz L, Brown EJ, Ullrich A, Bühring HJ (1999) Human signal-regulatory protein is expressed on normal, but not on subsets of leukemic myeloid cells and mediates cellular adhesion involving its counterreceptor CD47. Blood 94:3633-3643.

Silverman MA, Kaech S, Jareb M, Burack MA, Vogt L, Sonderegger P, Banker G (2001) Sorting and directed transport of membrane proteins during development of hippocampal neurons in culture. Proc Natl Acad Sci USA 98:7051-7057.
Tada K, Tanaka M, Hanayama R, Miwa K, Shinohara A, Iwamatsu A, Nagata S (2003) Tethering of apoptotic cells to phagocytes through binding of CD47 to src homology 2 domain-bearing protein tyrosine phosphatase substrate-1. J Immunol 171:5718-5726.

Tanaka H, Shan W, Phillips GR, Arndt K, Bozdagi O, Shapiro L, Huntley GW, Benson DL, Colman DR (2000) Molecular modification of N-cadherin in response to synaptic activity. Neuron 25:93-107.

Tienari PJ, De Strooper B, Ikonen E, Simons M, Weidemann A, Czech C Hartmann T, Ida N, Multhaup G, Masters CL, Van Leuven F, Beyreuther $\mathrm{K}$, Dotti CG (1996) The $\beta$-amyloid domain is essential for axonal sorting of amyloid precursor protein. EMBO J 15:5218-5229.

Vernon-Wilson EF, Kee WJ, Willis AC, Barclay AN, Simmons DL, Brown $\mathrm{MH}$ (2000) CD47 is a ligand for rat macrophage membrane signal regulatory protein SIRP $(\mathrm{OX} 41)$ and human $\operatorname{SIRP} \alpha 1$. Eur J Immunol 30:2130-2137

Wang XX, Dangott LJ, Pfenninger KH (2003) The heterogeneous growth cone glycoprotein gp93 is identical to the signal regulatory protein $\operatorname{SIRP} \alpha$ / SHPS-1/BIT. J Neurochem 86:55-60.

Williams AF, Barclay AN (1988) The immunoglobulin superfamily-domains for cell surface recognition. Annu Rev Immunol 6:381-405.

Winckler B, Mellman I (1999) Neuronal polarity: controlling the sorting and diffusion of membrane components. Neuron 23:637-640.

Yamao T, Matozaki T, Amano K, Matsuda Y, Takahashi N, Ochi F, Fujioka Y, Kasuga M (1997) Mouse and human SHPS-1: molecular cloning of cDNAs and chromosomal localization of genes. Biochem Biophys Res Commun 231:61-67. 